\section{Retinal nerve fibre layer measurements are reduced in patients with obstructive sleep apnoea syndrome}

\begin{abstract}
Purpose To determine the retinal nerve fibre layer (RNFL) thickness in patients with obstructive sleep apnoea syndrome (OSAS) in order to investigate the possibility of detecting early signs of glaucoma in this population.
\end{abstract}

Methods A total of 66 consecutive patients admitted for polysomnographic evaluation of suspected OSAS. Patients underwent an overnight sleep study in an effort to diagnose and determine the severity of OSAS. Patients who had the disease were classified as having mild and severe OSAS, while patients who did not have the disease were classified as controls. All patients received physical, neurological, and ophthalmological evaluation including visual acuity, slit-lamp examination, Goldmann applanation tonometry, gonioscopy with a three mirror contact lens, and fundus examination. After these examinations, patients with glaucoma and patients who had ophthalmological and/or systemic disease known to affect RNFL thickness were excluded from the study. The RNFL thickness was assessed with a scanning laser polarimeter (Nerve Fiber Analyzer GDx, Laser Diagnostic Technologies Inc., San Diego, CA, USA).

Results A total of 34 patients with obstructive sleep apnoea (19 mild, 15 severe) and 20 age-matched controls were included in the study. The thickness of RNFL was reduced in patients with OSAS compared to controls. The decrease in RNFL was found to be correlated with the severity of sleep apnoea $(r=0.78, P=0.01)$.

Conclusions The sleep apnoea syndrome is correlated with a proportional decrease in the RNFL. Decreased ocular perfusion related to hypoxia and vasospasm associated with OSAS
SH Kargi', R Altin², M Koksal' ${ }^{1}$ L Kart²,

$\mathrm{F} \mathrm{Cinar}^{3}$, SH Ugurbas ${ }^{1}$ and F Ayoglu ${ }^{4}$

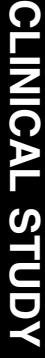

may cause RNFL thinning, which may precede clinically detectable glaucoma.

Eye (2005) 19, 575-579. doi:10.1038/sj.eye.6701582

Published online 27 August 2004

Introduction

Obstructive sleep apnoea syndrome (OSAS) is characterized by recurrent partial or complete upper airway obstruction during sleep. Several episodes that lead to hypoxia and hypercapnia may occur each night and cause sleep disruption and daytime sleepiness. ${ }^{1}$

OSAS is associated with optic neuropathy and glaucoma. ${ }^{2-6} \mathrm{~A}$ high glaucoma prevalance of $7.2 \%$ was detected in patients with OSAS. ${ }^{3}$ Additionally, patients with primary open-angle glaucoma or normal-tension glaucoma constitute a high-risk population for sleepapnoea syndrome. ${ }^{5-7}$

Optic neuropathy associated with glaucoma is characterized by increased size of the optic nerve cup and associated thinning of retinal nerve fibre layer (RNFL). ${ }^{8}$ Signs of glaucoma first occur with thinning of the RNFL followed by progression of optic disc excavation. This is followed by characteristic visual field defects. ${ }^{9}$ Patients with glaucoma can lose $40 \%$ of retinal ganglion cell axons before a measurable visual defect is evident. ${ }^{10}$ Early detection of RNFL thinning offers an opportunity to detect glaucoma at its earlier stages. 8,9

Scanning laser polarimeter is a computerized image analysing technique designed for objective, quantitative in vivo measurement of the RNFL thickness. It accomplishes this by measuring the phase shift (retardation) of polarized laser light traversing the RNFL. ${ }^{8,11}$ This technique has been shown to be effective in differentiating eyes with glaucoma from normal
${ }^{1}$ Department of

Ophthalmology

School of Medicine

Karaelmas University

Zonguldak, Turkey

${ }^{2}$ Department of Pulmonary

Disease

School of Medicine

Karaelmas University

Zonguldak, Turkey

${ }^{3}$ Department of Ear Nose and Throat

School of Medicine

Karaelmas University

Zonguldak, Turkey

${ }^{4}$ Department of Public

Health

School of Medicine

Karaelmas University

Zonguldak, Turkey

Correspondence:

SH Kargi,MD

Bahcelievler Mah. Funda Sk. 4/10

Zonguldak 67100

Turkey

Tel: + 903722610169

Fax: +90372 2610155

E-mail: sebnemkargi@

yahoo.com

Received: 11 January 2004 Accepted: 18 March 2004 Published online: 27 August 2004 
eyes. ${ }^{8,12-14}$ It can also provide early evidence for RNFL thinning in subjects at risk for glaucoma with a sensitivity of $96 \%$ and a specifity of $93 \% .{ }^{15}$

Since OSAS has been associated with glaucoma, we performed scanning laser polarimetry in patients with OSAS to determine if we can detect RNFL thinning in OSAS, and we compared the findings with control eyes.

\section{Materials and methods}

Of 72 patients consecutively admitted for polysomnographic evaluation of suspected OSAS, 66 patients agreed to have an ophthalmological examination. Informed consent was obtained from all patients. All patients underwent a overnight sleep study in a sleep laboratory in an effort to diagnose and assign a degree of severity for the OSAS. Apnoea information was gathered with a POLY-MESAM (MAP; Martinsried, Germany) unit consisting of a flow sensor for nasal and oral breath flow, laryngeal microphone, and three-channel ECG. Data were stored and the apnoea-hypopnea index (AHI), the apnoea index (AI), hypopnea index $(\mathrm{HI})$, and the oxygen desaturation index (ODI) were calculated.

The sum of apnoea and hypopnea episodes per hour during the night for each patient determined the apnoeahypopnea index and patients with an $\mathrm{AHI} \geqslant 5$ detected by sleep study were considered as having OSAS. These patients were seperated into two groups: Group 1 (mild, $\mathrm{AHI}=6-20$ ) and Group 2 (severe, AHI $\geqslant 20) .{ }^{16}$ Patients who had an apnoea-hypopnea index $<5$ were accepted as normal.

All patients received physical, neurological, and ophthalmologic evaluation including best-corrected visual acuity, slit-lamp examination, Goldmann applanation tonometry, gonioscopy with a three mirror contact lens, and fundus examination after pupil dilation. Automated visual field examination was performed in selected patients with the Humphrey Field Analyzer (Humphrey Systems, Inc., Dublin, CA, USA). At the time of ophthalmological examination, the results of the work-up for OSAS by sleep study were unknown to the examiner and the patient.

After ophthalmologic and systemic examinations, patients who had glaucoma, glaucomatous optic nerve cupping and glaucomatous visual field defects were excluded from the study. The following criteria were used for inclusion: (1) best-corrected visual acuity of $6 / 6$ or better, (2) intraocular pressure of $21 \mathrm{mmHg}$ or lower, (3) open angles by gonioscopy, (4) normal appearance of the optic nerve head by stereobiomicroscopy, (5) absence of any history suggestive of increased IOP, ocular trauma, or any family history of glaucoma, and (6) absence of any systemic diseases other than OSAS.
In all, 12 patients were excluded from the study as they did not fulfil the above criteria. Of these 12 patients, seven had OSAS and the other five did not have OSAS. The reasons for exclusion from the study were as follows: one patient was found to have primary open-angle glaucoma and two patients were found to have normaltension glaucoma, while one patient was previously diagnosed with primary open-angle glaucoma. One patient had corneal scarring secondary to ocular trauma and an other had degenerative myopia. Five patients had systemic hypertension and one patient had diabetes mellitus.

In all, 34 patients in the study and 20 patients in the control group were included and underwent RNFL measurements. The RNFL thickness was measured by the same ophthalmologist (SHK) using the GDx Nerve fibre Analyzer (Laser Diagnostic Technologies Inc., San Diego, CA, USA). A total of 65536 retinal locations were measured to create a map of RNFL thickness over a $15^{\circ}$ retinal area. Three measurements were taken for each eye and a composite image was created. The optic disc margin was outlined by a circle or ellipse placed around the inner margin of the peripapillary scleral ring by the operator. A measuring circle or ellipse was then generated by the instrument at 1.75 disc diameters concentric with the margin of the disc. For regional analysis of the RNFL thickness, the image was divided into four quadrants supplied by the manufacturer: superior $\left(120^{\circ}\right)$, nasal $\left(70^{\circ}\right)$, inferior $\left(120^{\circ}\right)$, and temporal $\left(50^{\circ}\right)$. The GDx 'extended analysis' program automatically calculated 16 RNFL measurements in each of the four quadrants and nine of these were used in this study. The nerve fibre layer parameters are defined in Table 1.

Statistical analysis was carried out using SPSS for Windows (version 11.0). Kruskal-Wallis analysis of variance test was used to compare the RNFL parameters among the groups. If there were any significant differences among the groups as determined by the analysis of variance, then Mann-Whitney $U$ test was used to evaluate differences between groups. The association between nerve fibre layer measurements and apnoea-hypopnea index was evaluated by the Pearson correlation coefficient $r$. A probability value $P$ of less than or equal to 0.05 was considered statistically significant.

\section{Results}

In all, 34 patients with OSAS (19 mild and 15 severe) and 20 controls were chosen for the study. The features of patients are summarized in Table 2. There were no differences between the groups with regard to gender, race, and age. The mean $\mathrm{O}_{2}$ saturation (the average value of oxygen saturations calculated for each case throughout 
Table 1 Nerve Fiber Analyzer Standard Parameters

\begin{tabular}{|c|c|}
\hline GDx parameter & Definition \\
\hline Symmetry & $\begin{array}{l}\text { Ratio of the average of the } 1500 \text { thickest pixels in the superior quadrant to the average } 1500 \\
\text { thickest pixels in the inferior quadrant }\end{array}$ \\
\hline Superior (inferior) ratio & $\begin{array}{l}\text { Ratio of the } 1500 \text { thickest pixels in the superior (inferior) quadrant over the average of the } 1500 \\
\text { median pixels in the temporal quadrant }\end{array}$ \\
\hline Superior-nasal ratio & $\begin{array}{l}\text { Ratio of the } 1500 \text { thickest pixels in the superior quadrant to the average of the } 1500 \text { median } \\
\text { pixels in the nasal quadrant }\end{array}$ \\
\hline Maximum modulation & Indicator of difference between the thickest and the thinnest areas of the image \\
\hline Ellipse modulation & Indicator of difference between the thickest and the thinnest areas of the ellipse \\
\hline Superior (inferior) average & $\begin{array}{l}\text { Average NFL thickness beneath the portion of the ellipse surrounding the optic nerve in the } \\
\text { superior (inferior) quadrant, in microns }\end{array}$ \\
\hline Ellipse average & Average NFL thickness beneath the ellipse \\
\hline
\end{tabular}

Table 2 Patient demographics with obstructive sleep apnoea syndrome and controls

\begin{tabular}{|c|c|c|c|c|c|}
\hline & \multicolumn{2}{|c|}{ Obstructive sleep apnoea syndrome } & \multirow[t]{2}{*}{ Control } & \multirow[t]{2}{*}{ P-value ${ }^{a}$} & \multirow[t]{2}{*}{ P-value } \\
\hline & $\begin{array}{l}\text { Group } 1 \text { (mild) } \\
(A H I<20)\end{array}$ & $\begin{array}{c}\text { Group } 2 \text { (severe) } \\
(A H I \geqslant 20)\end{array}$ & & & \\
\hline Age (years) & $45.1 \pm 6.4$ & $46.0 \pm 8.7$ & $43.68 \pm 6.12$ & NS & NS \\
\hline Number & 19 & $1 \overline{5}$ & 20 & & \\
\hline \multicolumn{6}{|l|}{ Sex } \\
\hline Male & 14 & 14 & 16 & NS & NS \\
\hline Female & 5 & 1 & 4 & & \\
\hline Mean oxygen saturation $(\%)$, mean \pm SD & $92.8 \pm 2.1$ & $90.2 \pm 4.2$ & $96.7 \pm 1.0$ & $<0.05$ & $<0.001$ \\
\hline Lowest saturation $(\%)$, mean $\pm \mathrm{SD}$ & $80.8 \pm 6.0$ & $68.9 \pm 8.5$ & $89.7 \pm 2.44$ & $<0.01$ & $<0.001$ \\
\hline
\end{tabular}

${ }^{\mathrm{a} C}$ Corresponding pairs: Group 1 and control, $t$-test.

${ }^{\mathrm{b}}$ Corresponding pairs: Group 2 and control, $t$-test.

NS: Statistically nonsignificant values.

the night) and lowest saturation (the lowest $\mathrm{O}_{2}$ saturation value calculated throughout the night for each case) were compared and both were found to be lower in patients with severe OSAS.

The distribution of RNFL measurement parameters calculated by GDx the Nerve Fibre Analyzer is given in Table 3. The mean values of all parameters except ellipse modulation were different between control and patient groups. All parameters were significantly decreased in patients with severe OSAS compared to controls $(P<0.05)$, indicating diffusely decreased RNFL thickness. Parameters of 'symmetry', 'superior ratio', and 'maximum modulation' were statistically different between controls and mild OSAS group $(P<0.05)$. RNFL measurements that showed statistically different values between mild and severe OSAS groups were seen in the 'ellipse average', 'superior average', and 'inferior average' $(P<0.05)$. The apnoea-hypopnea index was strongly correlated with a decrease in the RNFL ( $r=0.78, P=0.01)$.

\section{Discussion}

Obstructive sleep apnoea is a risk factor for cardiovascular and neurovascular diseases. ${ }^{3}$
During sleep, repetitive episodes of airway occlusion, with consequent hypoxaemia, hypercapnia, and changes in intrathoracic pressure elicit changes in the autonomic, haemodynamic, humoral, and neurorendocrine responses that can affect the circulation of the optic nerve with loss of ganglion cells. ${ }^{17}$

Progressive decrease in the RNFL thickness occurs in glaucoma. This thinning can be present in eyes of patients with glaucoma before detectable changes occur in the visual field. ${ }^{10}$ If a decrease in the RNFL can be reliably detected, the clinician may be alerted to the risk for developing glaucoma. ${ }^{8,9}$ To the best of our knowledge, there have not been any reports evaluating RNFL thickness in patients with OSAS, even though an association between glaucoma and sleep apnoea syndrome has been reported..$^{3-6}$ In this study, we measured the RNFL thickness to assess retinal nerve fibre injury reflected by thinning of this layer in OSAS. We found that the RNFL was thinner in patients with OSAS without any evident glaucoma as patients with clinical signs of glaucoma were excluded from the study. Additionally, RNFL thinning in OSAS was correlated with the severity of this condition.

RNFL measurement with the Nerve fibre Analyzer has been used to differentiate patients with glaucoma or 
Table 3 Nerve Fiber Analyzer parameters among patients with mild OSAS (Group 1), severe OSAS (Group 2) and controls: mean \pm SD

\begin{tabular}{|c|c|c|c|c|c|c|c|}
\hline & $\begin{array}{l}\text { Control } \\
(\mathrm{n}=20)\end{array}$ & $\begin{array}{l}\text { Group } 1 \\
(\mathrm{n}=19)\end{array}$ & $\begin{array}{c}\text { Group } 2 \\
(\mathrm{n}=15)\end{array}$ & P-Value ${ }^{a}$ & P-Value ${ }^{b}$ & P-Value ${ }^{c}$ & P-Value ${ }^{d}$ \\
\hline Symmetry & $1.0 \pm 0.1$ & $0.9 \pm 0.1$ & $0.9 \pm 0.1$ & 0.003 & 0.001 & 0.012 & 0.706 \\
\hline Superior ratio & $2.9 \pm 0.4$ & $2.5 \pm 0.6$ & $2.4 \pm 0.3$ & 0.001 & 0.012 & 0.001 & 0.656 \\
\hline Inferior ratio & $2.9 \pm 0.6$ & $2.7 \pm 0.6$ & $2.3 \pm 0.4$ & 0.01 & 0.226 & 0.005 & 0.118 \\
\hline Superior-nasal ratio & $2.4 \pm 0.4$ & $2.2 \pm 0.3$ & $1.9 \pm 0.2$ & 0.002 & 0.267 & 0.001 & 0.087 \\
\hline Maximum modulation & $2.1 \pm 0.5$ & $1.8 \pm 0.5$ & $1.6 \pm 0.4$ & 0.003 & 0.025 & 0.001 & 0.336 \\
\hline Ellipse modulation & $3.0 \pm 0.6$ & $2.9 \pm 0.8$ & $2.7 \pm 0.7$ & 0.159 & 0.592 & 0.05 & 0.167 \\
\hline Superior average & $90.5 \pm 10.4$ & $83.8 \pm 13.4$ & $73.4 \pm 8.8$ & 0.001 & 0.099 & 0.001 & 0.004 \\
\hline Inferior average & $90.7 \pm 10.3$ & $90.9 \pm 14.8$ & $76.8 \pm 10.6$ & 0.002 & 0.979 & 0.001 & 0.002 \\
\hline Ellipse average & $78.1 \pm 8.4$ & $74.9 \pm 13.4$ & $61.9 \pm 7.4$ & 0.001 & 0.196 & 0.001 & 0.004 \\
\hline
\end{tabular}

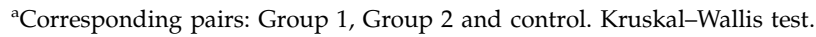

${ }^{\mathrm{b} C}$ Corresponding pairs: Group 1 and control. Mann-Whitney U test.

${ }^{\text {cC}}$ Corresponding pairs: Group 2 and control. Mann-Whitney U test.

${ }^{\mathrm{d} C}$ Corresponding pairs: Group 1 and Group 2. Mann-Whitney U test.

glaucoma suspects from the healthy controls..$^{8,12-14}$ Standard ratio-based or modulation parameters are calculated by the instrument after defining RNFL in different regions. The most reliable and sensitive measurement parameters used to differentiate glaucoma from healthy controls differs between reports. ${ }^{8,12,14}$ Recent studies have attempted to determine the correlation of RNFL thickness with visual field defects to correlate the most important parameters reflecting glaucoma. The RNFL thickness has been shown to be decreased in the corresponding quadrant even when the visual field was still normal in patients with glaucoma. ${ }^{11,18,19}$ The RNFL was found to be thinner and correlated with visual field loss in the superior and inferior quadrants. ${ }^{20,21}$ It has been suggested and experience would support that the nasal and temporal RNFL measurements when used for the ratios may be more helpful at early stages as the thickness of the superior and inferior quadrants is affected earlier in glaucoma. ${ }^{8}$ Kwon et $a l^{11}$ found that the number, deviation from normal for superior and inferior quadrants, ellipse average, and ellipse modulation were the most important parameters that were correlated with visual field defects. Ellipse modulation reported to be the most important parameter for differentiating glaucoma and maximum modulation showed stronger correlation with fields. . $^{12,22}$

The RNFL measurement parameters used in our study are chosen from the parameters that were previously found to be correlated with glaucomatous visual field defects. We found that almost all of the nerve fibre layer parameters were decreased in patients with OSAS, which suggests that there is a diffuse loss of axons entering the neuroretinal rim as expected in glaucoma. The ratios in which the superior and inferior RNFL thickness are divided for the RNFL thickness in the nasal and temporal quadrants that were previously found to correlate with visual field defects were also decreased in patients with OSAS.

With the increasing severity of OSAS, mean oxygen saturation values are reduced and relative hypoxia occurs. We found that, as the disease becomes more severe, RNFL thinning is proportionally more prominent. This supports the correlation found between hypoxia and RNFL thinning in our study.

Glaucomatous optic nerve damage has lately been considered to be the result of vascular and other pathogenic mechanisms in addition to elevated intraocular pressure. ${ }^{5,23}$

We propose two possible mechanisms for RNFL thinning in OSAS. We speculate that the first mechanism may be due to the following sequence: the sleeping state in OSAS is associated with a reduction in the ventilatory drive caused by hypoxia and hypercapnia. This causes a decrease in $\mathrm{pO}_{2}$ and an increase in $\mathrm{pCO}_{2} \cdot{ }^{1}$ Hypoxaemia results in increased levels of the vasoconstrictor endothelin production. The endothelial cells also produce nitric oxide, a vasodilator. ${ }^{24,25}$ In patients with OSAS, the endothelium-mediated vasodilator response is markedly impaired. Thinning of RNFL may be caused by a loss of ganglion cells caused in some way by the hypoxia secondary to this OSAS-induced imbalance between nitric oxide and endothelin. ${ }^{24-26}$

Our second speculation is that the nocturnal vascular changes caused by OSAS may be the cause of RNFL thinning. Hypoxia indirectly increased intracranial pressure during sleep and decreased cerebral perfusion pressure may disturb blood supply of the optic nerve in patients with OSAS. ${ }^{2,6,27,28}$ Orbital blood flow measured by colour Doppler ultrasonography was found to be higher in patients with OSAS compared to controls and increase in blood flow was accepted as a vasodilatory response to hypercapnia, one of the most important 
consequences of OSAS. ${ }^{29}$ Vascular disturbances may result with diffuse loss or localized defects of the RNFL before initation of glaucoma. ${ }^{30}$ When vascular dysregulation in OSAS is added to nocturnal systemic hypotension, RNFL damage and consequent thinning may occur.

We speculate that profound hypoxia and vascular dysregulation in OSAS may be the cause of RNFL thinning by disturbing ocular perfusion. The dysregulation of optic nerve circulation seen in OSAS may cause changes similar to glaucoma in the RNFL. Patients suspected with glaucoma should be screened for OSAS. Patients with OSAS may benefit by detecting RNFL thinning, which may provide early detection of optic neuropathy before glaucoma changes become manifest on visual field testing.

\section{References}

1 Phillipson EA. Sleep disorders. In: Murray JF, Nadel JA (eds). Textbook of Respiratory Medicine. WB Saunders: Philadelphia, 1994, pp 2301-2304.

2 Mojon DS, Mathis J, Zulauf M, Koerner F, Hess CW. Optic neuropathy associated with sleep apnea syndrome. Ophthalmology 1998; 105: 874-877.

3 Mojon DS, Hess CW, Goldblum D, Fleischhauer J, Koerner F, Bassetti C et al. High prevalence of glaucoma in patients with sleep apnea syndrome. Ophthalmology 1999; 106: 1009-1012.

4 Mojon DS, Hess CW, Goldblum D, Fleischhauer J, Bohnke M, Korner F. Sleep apnea syndrome is associated with glaucoma. Ophthalmic Res 1998; 30S1: 147.

5 Mojon DS, Hess CW, Goldblum D, Bohnke M, Korner F, Mathis J. Primary open angle glaucoma is associated with sleep apnea syndrome. Ophthalmologica 2000; 214: 115-118.

6 Mojon DS, Hess CW, Goldblum D, Boehnke M, Koerner F, Gugger $\mathrm{M}$ et al. Normal-tension glaucoma is associated with sleep apnea syndrome. Ophthalmologica 2002; 216: 180-184.

7 Goldblum D, Mathis J, Bohnke M, Bassetti C, Hess CW, Gugger $\mathrm{M}$ et al. Nocturnal measurements of intraocular pressure in patients with normal-tension glaucoma and sleep apnea syndrome. Klin Monatsbl Augenheilkd 2000; 216: 246-249.

8 Lee VW, Hei Mok K. Retinal nerve fibre layer measurement by nerve fibre analyzer in normal subjects and patients with glaucoma. Ophthalmology 1999; 106: 1006-1008.

9 Kremmer S, Ayertey HD, Selbach JM, Steuhl KP. Scanning laser polarimetry, retinal nerve fibre layer photography, and perimetry in the diagnosis of glaucomatous nerve fibre defects. Graefes Arch Clin Exp Opht 2000; 238: 922-926.

10 Quigley HA, Dunkelberger GR, Green WR. Retinal ganglion cell atrophy correlated with automated perimetry in human eyes with glaucoma. Am J Ophthalmol 1989; 107: 453-464.

11 Kwon YH, Hong S, Honkanen RA, Alward WLM. Correlation of automated visual field parameters and peripapillary nerve fibre layer thickness as measured by scanning laser polarimetry. J Glaucoma 2000; 9: 281-288.

12 Choplin NT, Lundy DC, Dreher AW. Differentiating patients with glaucoma from glaucoma suspects and normal subjects by nerve fibre layer assessment with scanning laser polarimetry. Ophthalmology 1998; 105: 2068-2076.
13 Choplin NT, Lundy DC. The sensitivity and specificity of scanning laser polarimetry in the detection of glaucoma in a clinical setting. Ophthalmology 2001; 108: 899-904.

14 Weinreb RN, Zangwill L Berry CC, Bathija R, Sample PA. Detection of glaucoma with scanning laser polarimetry. Arch Ophthalmol 1998; 116: 1583-1589.

15 Tjon-Fo-Sang MJ, Lemij HG. The sensitivity and specifity of nerve fibre layer measurements in glaucoma as determined with scanning laser polarimetry. Am J Ophthalmol 1997; 123: 62-69.

16 Polysomnography Task Force American Sleep Disorders Association of Practice Committee. Practice parameters for the indications for polysomnography and related procedures. Sleep 1997; 20: 406-422.

17 Lanfranchi P, Somers VA. Obstructive sleep apnea and vascular disease. Respir Res 2001; 2: 315-319.

18 Matsumoto C, Shirato S, Haneda M, Yamashiro H, Saito M. Study of retinal nerve fibre layer thickness within normal hemivisual field in primary open-angle glaucoma and normal-tension glaucoma. Jpn J Ophthalmol 2003; 47: 22-27.

19 Reyes RDC, Tomita G, Kitazawa Y. Retinal nerve fibre layer thickness within the area of apparently normal visual field in normal-tension glaucoma with hemifield defect. J Glaucoma 1998; 7: 329-335.

20 Matsuno K, Kurimoto Y, Umihira J, Hoya T, Yoshimura N. Comparative study of retinal nerve fibre layer loss in normal-tension glaucoma and chronic open-angle glaucoma. Ophthalmologica 2001; 215: 108-112.

21 Weinreb RN, Shakiba S, Sample PA, Shahrokni S, van Horn S, Garden VS et al. Association between quantitative nerve fibre layer measurement and visual field loss in glaucoma. Am J Ophthalmol 1995; 120: 732-738.

22 Chen YY, Chen PP, Xu L, Ernst PK, Wang L, Mill RP. Correlation of peripapillary nerve fibre layer thickness by scanning laser polarimetry with visual field defects in patients with glaucoma. J Glaucoma 1998; 7: 312-316.

23 Hayreh SS. The role of age and cardiovascular disease in glaucomatous optic neuropathy. Surv Ophthalmol 1999; 43 (Suppl 1): S27-S42.

24 Kourembanas S, Marsden PA, McQuillan LP, Faller DV. Hypoxia induces endothelin gene expression and secretion in cultured human endothelium. J Clin Invest 1991; 88: 1054-1057.

25 Kato M, Roberts-Thomson P, Phillips BG, Haynes WG, Winnicki M, Accurso V et al. Impairment of endotheliumdependent vasodilation of resistance vessels in patients with obstructive sleep apnea. Circulation 2000; 102: 2607-2610.

26 Narkiewicz K, Montano N, Cogliati C, van de Borne PJ, Dyken ME, Somers VK. Altered cardiovascular variability in obstructive sleep apnea. Circulation 1998; 98: 1071-1077.

27 Jennum P, Borgesen SE. Intracranial pressure and obstructive sleep apnea. Chest 1989; 95: 279-283.

28 Bucci Jr FA, Krohel GB. Optic nerve swelling secondary to the obstructive sleep apnea syndrome. Am J Ophthalmol 1988; 105: 428-430.

29 Erdem CZ, Altin R, Erdem LO, Kargi S, Kart L, Cinar F, Ayoglu F. Doppler measurements of blood flow velocities in extraocular orbital vessels in patients with obstructive sleep apnea syndrome. J Clin Ultrasound 2003; 31: 250-257.

30 Hayreh SS, Jonas JB. Appearance of the optic disk and retinal nerve fibre layer in atherosclerosis and arterial hypertension: an experimental study in rhesus monkeys. Am J Ophthalmol 2000; 130: 91-96. 